Supporting Information

\title{
The Application of C-H Functionalization in the
}

\section{Development of a Concise and Convergent Route to}

\section{the Phosphatidylinositol-3-kinase Delta Inhibitor}

\section{Nemiralisib.}

Robert N. Bream, ${ }^{+}$Hugh Clark, Dean Edney, ${ }^{\dagger}$ Antal Harsanyi, ${ }^{\dagger}$ John Hayler, Alan

Ironmonger*, Nadine Mc Cleary, ${ }^{\dagger}$ Natalie Phillips, Catherine Priestley, Alastair Roberts,

Philip Rushworth, Peter Szeto, Michael R. Webb, and Katherine Wheelhouse*

Chemical Development, GlaxoSmithKline Medicines Research Centre, Gunnels Wood

Road, Stevenage, SG1 2NY, United Kingdom.

† R.N.B., D.E., N.M.C. and A.H. are former GSK employees. 
Synthetic methods and characterization data for compounds $3,17-26,29,32,37,4-$

chloro-1H-indazole and 44.

General Methods. All commercially-sourced chemicals and solvents were used as received without further purification. ${ }^{1} \mathrm{H}$ and ${ }^{13} \mathrm{C}$ NMR spectra were acquired on a Bruker AV-400 NMR spectrometer and chemical shifts are reported in parts per million relative to tetramethylsilane. High resolution mass spectra were recorded on a linear ion trap combined with a Fourier transform ion cyclotron resonance mass spectrometer using an electrospray ionization source operated in positive ion mode. Observed $\mathrm{m} / \mathrm{z}$ values and empirical formulae refer to $[\mathrm{M}+\mathrm{H}]^{+}$. Melting points were recorded using an automated melting point apparatus.<smiles>CCOC(=O)c1cnco1</smiles>

\section{Ethyl oxazole ethyl oxazole-5-carboxylate (3)}

A solution of ethyl glyoxalate (50 wt\% solution in PhMe, $20.0 \mathrm{ml}, 101 \mathrm{mmol}$ ) and DBU (12.4 ml, $82 \mathrm{mmol})$ in DCM (62 mL) was added over $1 \mathrm{hr}$ via cannula to a solution of 
tosylmethylisocyanate $(12.3 \mathrm{~g}, 63.1 \mathrm{mmol})$ in $\mathrm{DCM}(62 \mathrm{~mL})$ at $0{ }^{\circ} \mathrm{C}$ and aged for a further $1 \mathrm{hr}$. The mixture was quenched into $2 \mathrm{M} \mathrm{HCl}(150 \mathrm{~mL})$. This mixture was then extracted with DCM $(2 \times 100 \mathrm{~mL})$ and the solvent removed in vacuo. The resulting yellow oil was distilled at reduced pressure $\left(12 \mathrm{mbar}, 105^{\circ} \mathrm{C}\right)$ to afford the title compound $(7.10 \mathrm{~g}, 50.3$ $\mathrm{mmol}, 80 \%)$ as a colourless oil.

${ }^{1} \mathrm{H} \operatorname{NMR}\left(\mathrm{CDCl}_{3}, 400 \mathrm{MHz}\right) \delta_{\mathrm{H}} 8.01(1 \mathrm{H}, \mathrm{s}), 7.77(1 \mathrm{H}, \mathrm{s}), 4.41(2 \mathrm{H}, \mathrm{q}, J=7.1 \mathrm{~Hz}), 1.40$ $(3 \mathrm{H}, \mathrm{t}, J=7.1 \mathrm{~Hz}) .{ }^{13} \mathrm{C} \mathrm{NMR}\left(\mathrm{CDCl}_{3}, 100 \mathrm{MHz}\right) \delta_{\mathrm{C}} 157.5,153.2,142.9,133.2,61.7,14.2$ HRMS $m / z$ calc'd for $\mathrm{C}_{6} \mathrm{H}_{8} \mathrm{NO}_{3}$ 142.0499, measured 142.0495 .

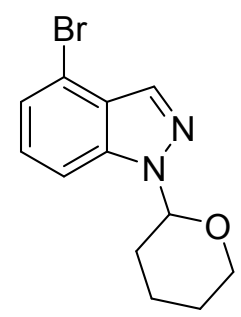

\section{4-Bromo-1-(tetrahydro-2H-pyran-2-yl)-1H-indazole (17)}


A mixture of 4-bromoindazole (25.3 g, $128 \mathrm{mmol})$, dihydropyran (40 mL, $438 \mathrm{mmol})$ and trifluoroacetic acid $(1.00 \mathrm{~mL}, 13.0 \mathrm{mmol})$ in EtOAc $(375 \mathrm{~mL})$, was heated at reflux for 20 hrs. The mixture was allowed to cool and the solvent removed in vacuo. The resulting oil was taken up in 2-propanol (100 mL) and heated to $55^{\circ} \mathrm{C}$ to afford a clear solution. The mixture was allowed to cool to $20^{\circ} \mathrm{C}$ resulting in precipitation of a crystalline solid, aged for $2 \mathrm{hrs}$, then filtered and washed with 2-propanol $(50 \mathrm{~mL})$ and dried in vacuo at $50^{\circ} \mathrm{C}$ to afford 4-bromo-1-(tetrahydro-2H-pyran-2-yl)-1H-indazole $(32.9 \mathrm{~g}, 117 \mathrm{mmol}, 91 \%)$ as an off-white solid.

${ }^{1} \mathrm{H}$ NMR $\left(400 \mathrm{MHz}, \mathrm{CDCl}_{3}\right) \delta_{\mathrm{H}} 8.04(1 \mathrm{H}, \mathrm{s}), 7.56(1 \mathrm{H}, \mathrm{d}, J=8.3 \mathrm{~Hz}), 7.33(1 \mathrm{H}, \mathrm{d}, J=7.4$ $\mathrm{Hz}), 7.24(1 \mathrm{H}, \mathrm{dd}, J=8.3,7.4 \mathrm{~Hz}), 5.72(1 \mathrm{H}, \mathrm{dd}, J=9.1,2.8 \mathrm{~Hz}), 4.06-3.98(1 \mathrm{H}, \mathrm{m}), 3.79-$ $3.71(1 \mathrm{H}, \mathrm{m}), 2.62-2.50(1 \mathrm{H}, \mathrm{m}), 2.23-2.04(2 \mathrm{H}, \mathrm{m}), 1.85-1.63(3 \mathrm{H}, \mathrm{m}) ;{ }^{13} \mathrm{C}$ NMR $(100$ $\left.\mathrm{MHz}, \mathrm{CDCl}_{3}\right) \delta_{\mathrm{C}} 140.1,133.8,127.3,125.8,124.0,114.5,109.4,85.7,67.3,29.3,25.0$ 22.4; HRMS $m / z$ calc'd for $\mathrm{C}_{12} \mathrm{H}_{14} \mathrm{BrN}_{2} \mathrm{O}_{1} 281.0284$, measured 281.0283; m.p. $75-78{ }^{\circ} \mathrm{C}$. 


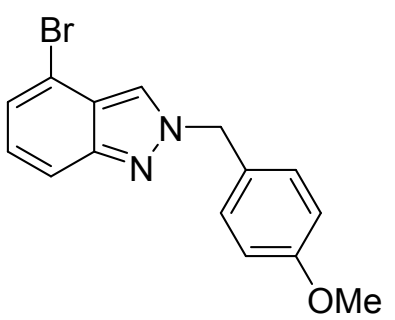

4-bromo-1-(4-methoxybenzyl)-1H-indazole

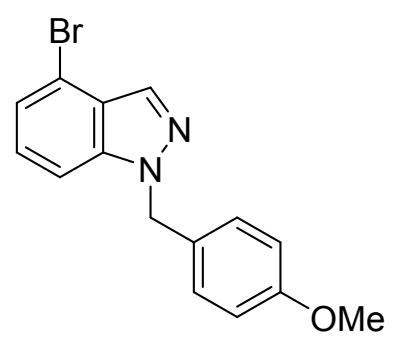

and 4-bromo-2-(4-methoxybenzyl)-2H-

indazole (18) and (19)

A mixture of 4-bromoindazole $(3 \mathrm{~g}, 15.2 \mathrm{mmol})$, tetrabutylammonium hydrogen sulfate

$(0.258 \mathrm{~g}, 0.761 \mathrm{mmol})$ and sodium hydroxide $(1.522 \mathrm{~g}, 38.1 \mathrm{mmol})$ was stirred in THF

$(9.9 \mathrm{~mL})$ at $20{ }^{\circ} \mathrm{C}$ for $1 \mathrm{hr}$. The resulting solution was cooled to $15{ }^{\circ} \mathrm{C}$ and paramethoxybenzyl chloride $(2.27 \mathrm{~mL}, 16.8 \mathrm{mmol})$ was added dropwise over 5 minutes. The reaction mixture was then stirred under a nitrogen atmosphere at $20^{\circ} \mathrm{C}$ for $16 \mathrm{hr}$ and then poured into a mixture of hydrochloric acid $(5.1 \mathrm{~mL}, 38.0 \mathrm{mmol})$ and water $(60.0 \mathrm{~mL})$. After stirring the oily suspension for 30 mins, a pale yellow suspension formed, which was filtered and washed with water, then dried at $50{ }^{\circ} \mathrm{C}$ in vacuo. The resulting mixture of compounds was purified by silica gel chromatography, eluting with $2-22 \%$ EtOAc in 
heptanes to afford 4-bromo-1-(4-methoxybenzyl)-1H-indazole (18) as a white solid (2.87 g,

$8.96 \mathrm{mmol}, 59 \%)$

${ }^{1} \mathrm{H} \mathrm{NMR}\left(400 \mathrm{MHz}, \mathrm{CDCl}_{3}\right) \delta_{\mathrm{H}} 8.04(1 \mathrm{H}, \mathrm{d}, J=0.7 \mathrm{~Hz}), 7.38(1 \mathrm{H}, \mathrm{d}, J=4.9,0.7 \mathrm{~Hz}), 7.36$

$(1 \mathrm{H}, \mathrm{t}, J=4.9 \mathrm{~Hz}), 7.24(2 \mathrm{H}, \mathrm{d}, J=8.8 \mathrm{~Hz}), 7.24(1 \mathrm{H}, \mathrm{d}, J=4.9 \mathrm{~Hz}), 6.91(2 \mathrm{H}, \mathrm{d}, J=8.8$

$\mathrm{Hz}), 5.60(2 \mathrm{H}, \mathrm{s}), 3.85(3 \mathrm{H}, \mathrm{s}) ;{ }^{13} \mathrm{C} \mathrm{NMR}\left(100 \mathrm{MHz}, \mathrm{CDCl}_{3}\right) \delta_{\mathrm{C}} 159.3,140.0,133.4,128.6$,

$128.4,127.1,125.5,123.5,114.7,114.2,108.5,55.2,53.0$; HRMS $\mathrm{m} / \mathrm{z}$ calc'd for

$\mathrm{C}_{15} \mathrm{H}_{14} \mathrm{BrN}_{2} \mathrm{O}$ 317.0284, measured 317.0287; m.p. $69-71{ }^{\circ} \mathrm{C}$; and 4-bromo-2-(4methoxybenzyl)-2H-indazole (19) as a white solid (0.94 g, $2.82 \mathrm{mmol}, 19 \%$ ).

${ }^{1} \mathrm{H}$ NMR $\left(400 \mathrm{MHz}, \mathrm{CDCl}_{3}\right) \delta_{\mathrm{H}} 7.87(1 \mathrm{H}, \mathrm{s}), 7.67(1 \mathrm{H}, \mathrm{d}, J=8.7 \mathrm{~Hz}), 7.29(2 \mathrm{H}, \mathrm{d}, J=8.8$

$\mathrm{Hz}), 7.23(1 \mathrm{H}, \mathrm{d}, J=7.1 \mathrm{~Hz}), 3.82(3 \mathrm{H}, \mathrm{s}), 7.14(1 \mathrm{H}, \mathrm{dd}, J=8.7,7.1 \mathrm{~Hz}), 6.91(2 \mathrm{H}, \mathrm{d}, J=$ $8.8 \mathrm{~Hz}), 5.53(2 \mathrm{H}, \mathrm{s}) ;{ }^{13} \mathrm{C} \mathrm{NMR}\left(100 \mathrm{MHz}, \mathrm{CDCl}_{3},\right) \delta_{\mathrm{C}} 159.9,148.9,129.7,127.2,126.6$, 124.3, 123.6, 116.8, 114.4, 114.2, 112.9, 57.3, 55.3; HRMS $m / z$ calc'd for $\mathrm{C}_{15} \mathrm{H}_{14} \mathrm{BrN}_{2} \mathrm{O}$ 317.0284, measured 317.0286; m.p. $131-134^{\circ} \mathrm{C}$.

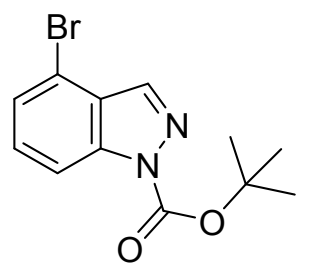




\section{tert-butyl 4-bromo-1 H-indazole-1-carboxylate (20)}

A mixture of 4-bromoindazole $(3.00 \mathrm{~g}, 15.2 \mathrm{mmol})$, di-tert-butylpyrocarbonate $(4.98 \mathrm{~g}$, $22.8 \mathrm{mmol})$ and $\operatorname{DMAP}(0.372 \mathrm{~g}, 3.05 \mathrm{mmol})$ was stirred in $\mathrm{MeCN}(30 \mathrm{ml})$ at $20{ }^{\circ} \mathrm{C}$ for 1 hr. The resulting suspension was poured into a stirred solution of hydrochloric acid (2.54 $\mathrm{mL}, 19.0 \mathrm{mmol})$ in water $(60 \mathrm{~mL})$ and stirred for 30 mins. The mixture was extracted with

$\operatorname{DCM}(2 \times 30 \mathrm{~mL})$ and the combined organic layers washed with water $(10 \mathrm{~mL})$ and evaporated to dryness. Purification by silica gel chromatography, eluting with $2-22 \%$ EtOAc in heptane, afforded tert-butyl 4-bromo-1H-indazole-1-carboxylate $(2.74 \mathrm{~g}, 9.22$ mmol, 61\%) as an off-white solid.

${ }^{1} \mathrm{H}$ NMR $\left(400 \mathrm{MHz}, \mathrm{CDCl}_{3}\right) \delta_{\mathrm{H}} 8.18(1 \mathrm{H}, \mathrm{d}, J=0.7 \mathrm{~Hz}), 8.15(1 \mathrm{H}, \mathrm{d}, J=8.3 \mathrm{~Hz}), 7.45(1 \mathrm{H}$, $\mathrm{dd}, J=7.6,0.7 \mathrm{~Hz}), 7.38(1 \mathrm{H}, \mathrm{dd}, J=8.3,7.6 \mathrm{~Hz}), 1.73(9 \mathrm{H}, \mathrm{s}) ;{ }^{13} \mathrm{C}$ NMR $(100 \mathrm{MHz}$, $\left.\mathrm{CDCl}_{3}\right) \delta_{\mathrm{C}} 148.9,140.5,139.0,129.7,126.8,126.5,114.5,113.6,85.4,28.1 ; \mathrm{HRMS} m / z$ calc'd for $\mathrm{C}_{12} \mathrm{H}_{13} \mathrm{BrN}_{2} \mathrm{O}_{2} \mathrm{Na} 319.0053$, measured 319.0061; m.p. 87-89 ${ }^{\circ} \mathrm{C}$.

Note: $\mathrm{MH}^{+}$not found, however, also $m / z$ calc'd for $\mathrm{C}_{8} \mathrm{H}_{6} \mathrm{BrN}_{2} \mathrm{O}_{2}\left(\mathrm{MH}^{+}\right.$- 2-butene) 240.9607, found 240.9612 and $m / z$ calc'd for $\mathrm{C}_{7} \mathrm{H}_{6} \mathrm{BrN}_{2}\left(\mathrm{MH}^{+}\right.$- 2-butene - $\left.\mathrm{CO}_{2}\right)$ 196.9709, found 196.9714. 


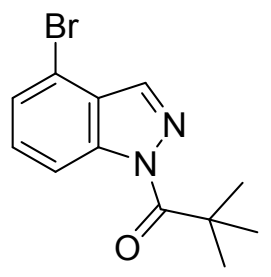

\section{1-(4-bromo-1H-indazol-1-yl)-2,2 dimethylpropan-1-one (21)}

To a mixture of 4-bromoindazole $(2.00 \mathrm{~g}, 10.2 \mathrm{mmol})$ and DMAP $(0.124 \mathrm{~g}, 1.02 \mathrm{mmol})$ stirred in THF (30.0 mL) at $20^{\circ} \mathrm{C}$ was added DIPEA $(3.55 \mathrm{~mL}, 20.3 \mathrm{mmol})$ followed by the dropwise addition of pivalic anhydride $(5.05 \mathrm{~mL}, 24.9 \mathrm{mmol})$ added over 5 minutes at $20^{\circ} \mathrm{C}$. The reaction solution was then heated to $70^{\circ} \mathrm{C}$ for $48 \mathrm{~h}$. Once complete consumption of starting material was observed, the mixture was cooled to $20{ }^{\circ} \mathrm{C}$ and aged for $72 \mathrm{~h}$. The resulting suspension was then added to a stirred solution of hydrochloric acid $(3.40 \mathrm{~mL}, 23.4 \mathrm{mmol})$ in water $(40 \mathrm{~mL})$. The suspension was aged for 30 mins then the aqueous layer was extracted with DCM $(2 \times 20 \mathrm{~mL})$. The combined organic layers were washed with water $(10 \mathrm{~mL})$ and then evaporated to dryness. The resulting crude product was purified by silica gel chromatography, eluting with 2-22\% EtOAc in heptanes to afford 1-(4-bromo-1H-indazol-1-yl)-2,2-dimethylpropan-1-one (2.53 g, $9.00 \mathrm{mmol}$ $89 \%)$ as a white solid.

${ }^{1} \mathrm{H}$ NMR $\left(400 \mathrm{MHz}, \mathrm{CDCl}_{3},\right) \delta_{\mathrm{H}} 8.43(1 \mathrm{H}, \mathrm{d}, J=8.4 \mathrm{~Hz}), 8.14(1 \mathrm{H}, \mathrm{d}, J=0.6 \mathrm{~Hz}), 7.49(1 \mathrm{H}$, dd, $J=7.6,0.6 \mathrm{~Hz}), 7.40(1 \mathrm{H}, \mathrm{dd}, J=8.4,7.6 \mathrm{~Hz}), 1.58(9 \mathrm{H}, \mathrm{s}) ;{ }^{13} \mathrm{C}$ NMR $(100 \mathrm{MHz}$, $\left.\mathrm{CDCl}_{3}\right) \delta_{\mathrm{C}} 178.5,141.2,138.2,127.1,126.3,2$ 115.2, 114.2, 42.0, 7.7; HRMS m/z calc'd for $\mathrm{C}_{12} \mathrm{H}_{14} \mathrm{BrN}_{2} \mathrm{O} 281.0284$, measured 281.0286 ; m.p. $130-131{ }^{\circ} \mathrm{C}$. 

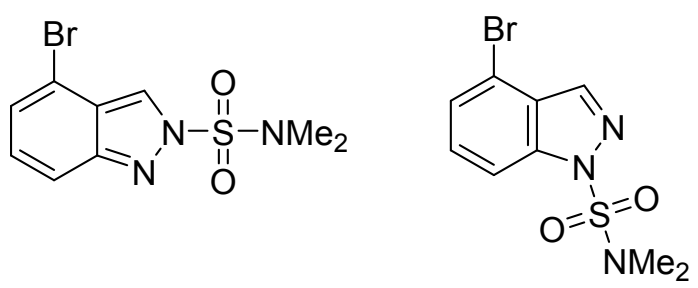

4-Bromo- $N, N$-dimethyl-1 $H$-indazole-1-sulfonamide (22) and 4-bromo- $N, N$-dimethyl-2 $H$ indazole-2-sulfonamide (23)

A mixture of 4-bromoindazole $(3.00 \mathrm{~g}, 15.2 \mathrm{mmol})$, tetrabutylammonium hydrogen sulfate $(0.258 \mathrm{~g}, 0.761 \mathrm{mmol})$ and sodium hydroxide $(1.52 \mathrm{~g}, 38.1 \mathrm{mmol})$ was stirred in THF $(9.9$ $\mathrm{mL}$ ) at $20{ }^{\circ} \mathrm{C}$ for $1 \mathrm{hr}$. The resulting solution was then cooled to $15{ }^{\circ} \mathrm{C}$ and $\mathrm{N}, \mathrm{N}$ dimethylaminosulfonyl chloride $(1.95 \mathrm{ml}, 18.3 \mathrm{mmol})$ was added dropwise over 5 minutes.

The reaction mixture was then stirred at $20^{\circ} \mathrm{C}$ for $1 \mathrm{hr}$ and then poured into a solution of hydrochloric acid $(2.54 \mathrm{~mL}, 19.0 \mathrm{mmol})$ in water $(21 \mathrm{~mL})$ and the resulting suspension aged for $1.5 \mathrm{hrs}$. The mixture was filtered and washed with water $(9 \mathrm{~mL})$, then dried in vacuo at $50{ }^{\circ} \mathrm{C}$. The resulting mixture of compounds was purified by silica gel chromatography, eluting with 2-22\% EtOAc in heptane to afford 4-bromo- $N, N$-dimethyl$1 /$-indazole-1-sulfonamide $(1.18 \mathrm{~g}, 3.88 \mathrm{mmol}, 26 \%)$ as a white solid 
${ }^{1} \mathrm{H}$ NMR $\left(400 \mathrm{MHz}, \mathrm{CDCl}_{3}\right) \delta_{\mathrm{H}} 8.22(1 \mathrm{H}, \mathrm{d}, J=0.5 \mathrm{~Hz}), 8.02(1 \mathrm{H}, \mathrm{d}, J=8.3 \mathrm{~Hz}), 7.47(1 \mathrm{H}$, $\mathrm{dd}, J=7.6,0.5 \mathrm{~Hz}), 7.38(1 \mathrm{H}, \mathrm{dd}, J=8.3,7.6 \mathrm{~Hz}), 3.00(6 \mathrm{H}, \mathrm{s}) ;{ }^{13} \mathrm{C}$ NMR $(100 \mathrm{MHz}$ $\left.\mathrm{CDCl}_{3}\right) \delta_{\mathrm{C}} 141.5,138.8,129.7,126.3,125.5,114.5,112.0,38.9 ; \mathrm{HRMS} \mathrm{m} / \mathrm{z}$ calc'd for $\mathrm{C}_{9} \mathrm{H}_{11} \mathrm{BrN}_{3} \mathrm{O}_{2} \mathrm{~S} 303.9750$ measured 303.9755 ; m.p. $96-98^{\circ} \mathrm{C}$; and 4-bromo- $N, N$-dimethyl2 H-indazole-2-sulfonamide ( $1.52 \mathrm{~g}, 4.95 \mathrm{mmol}, 33 \%)$ as a cream solid.

${ }^{1} \mathrm{H} \mathrm{NMR}\left(400 \mathrm{MHz}, \mathrm{CDCl}_{3}\right) \delta_{\mathrm{H}} 8.51(1 \mathrm{H}, \mathrm{d}, J=1.0 \mathrm{~Hz}), 7.68(1 \mathrm{H}, \mathrm{d}, J=8.8 \mathrm{~Hz}), 7.31(1 \mathrm{H}$, $\mathrm{dd}, J=7.1,1.0 \mathrm{~Hz}), 7.22(1 \mathrm{H}, \mathrm{dd}, J=8.8,7.1 \mathrm{~Hz}), 3.03(6 \mathrm{H}, \mathrm{s}) ;{ }^{13} \mathrm{C}$ NMR $(100 \mathrm{MHz}$, $\left.\mathrm{CDCl}_{3}\right) \delta_{\mathrm{C}} 150.3,129.1,126.3,126.2,122.8,117.8,113.7,38.9 ; \mathrm{HRMS} \mathrm{m} / z$ calc'd for $\mathrm{C}_{9} \mathrm{H}_{11} \mathrm{BrN}_{3} \mathrm{O}_{2} \mathrm{~S} 303.9750$, measured 303.9760; m.p. $151-154{ }^{\circ} \mathrm{C}$.

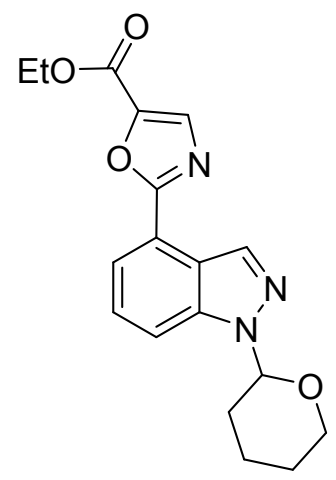

Ethyl 2-(1-(tetrahydro-2H-pyran-2-yl)-1 H-indazol-4-yl)oxazole-5-carboxylate (24)

4-Bromo-1-(tetrahydro-2H-pyran-2-yl)-1H-indazole (17) (8.8 g, $31.3 \mathrm{mmol}), \mathrm{Pd}(\mathrm{OAc})_{2}$

(351 mg, $1.57 \mathrm{mmol}), \mathrm{Cs}_{2} \mathrm{CO}_{3}(20.4 \mathrm{~g}, 62.6 \mathrm{mmol})$, pivalic acid $(1.28 \mathrm{~g}, 12.5 \mathrm{mmol})$ and 
dicyclohexyl(2',6'-diisopropoxy-[1,1'-biphenyl]-2-yl)phosphine (Ruphos, 1.46 g, 3.13

mmol) were combined in degassed PhMe (88 ml). Ethyl oxazole-5-carboxylate (3) (3.93

$\mathrm{ml}, 32.9 \mathrm{mmol}$ ) was added and the mixture heated at $100^{\circ} \mathrm{C}$ for $18 \mathrm{hrs}$. On cooling, the mixture was diluted with water $(90 \mathrm{~mL})$ and extracted with $\mathrm{PhMe}(2 \times 90 \mathrm{ml})$. The combined organics were evaporated to dryness. The resulting oil was triturated with $\mathrm{EtOH}$ (72 $\mathrm{mL}$ ) at $70{ }^{\circ} \mathrm{C}$ and the resultant slurry cooled to $20^{\circ} \mathrm{C}$, aged for $1 \mathrm{hr}$, filtered, washed with $\mathrm{EtOH}(20 \mathrm{~mL})$ and dried in vacuo at $50{ }^{\circ} \mathrm{C}$ to afford ethyl 2-(1-(tetrahydro-2 $\mathrm{H}$-pyran2-yl)-1 H-indazol-4-yl)oxazole-5-carboxylate $(9.50 \mathrm{~g}, 27.8 \mathrm{mmol}, 89 \%$ yield) as a white solid.

${ }^{1} \mathrm{H}$ NMR $\left(400 \mathrm{MHz}, \mathrm{CDCl}_{3}\right) \delta_{\mathrm{H}} 8.76(1 \mathrm{H}, \mathrm{s}), 8.04(1 \mathrm{H}, \mathrm{d}, J=7.3 \mathrm{~Hz}), 7.94(1 \mathrm{H}, \mathrm{s}), 7.79$ $(1 \mathrm{H}, \mathrm{d}, J=8.4 \mathrm{~Hz}), 7.50(1 \mathrm{H}, \mathrm{dd}, J=8.4,7.3 \mathrm{~Hz}), 5.80(1 \mathrm{H}, \mathrm{dd}, J=9.2,2.6 \mathrm{~Hz}), 4.44(2 \mathrm{H}$, $\mathrm{q}, J=7.1 \mathrm{~Hz}), 4.08-3.99(1 \mathrm{H}, \mathrm{m}), 3.82-3.73(1 \mathrm{H}, \mathrm{m}), 2.67-2.53(1 \mathrm{H}, \mathrm{m}), 2.24-2.08(2 \mathrm{H}$, $\mathrm{m}), 1.87-1.63(3 \mathrm{H}, \mathrm{m}), 1.44(3 \mathrm{H}, \mathrm{t}, J=7.1 \mathrm{~Hz}) ;{ }^{13} \mathrm{C}$ NMR $\left(100 \mathrm{MHz}, \mathrm{CDCl}_{3}\right) \delta_{\mathrm{C}} 163.3$ $157.9,142.2,139.9,135.5,134.3,126.2,121.6,121.6,119.0,113.8,85.6,61.5,67.4$ 29.4, 25.1, 22.4, 14.3; HRMS $m / z$ calc'd for $\mathrm{C}_{18} \mathrm{H}_{20} \mathrm{~N}_{3} \mathrm{O}_{4} 342.1448$, measured 342.1454. m.p. $140-142{ }^{\circ} \mathrm{C}$. 


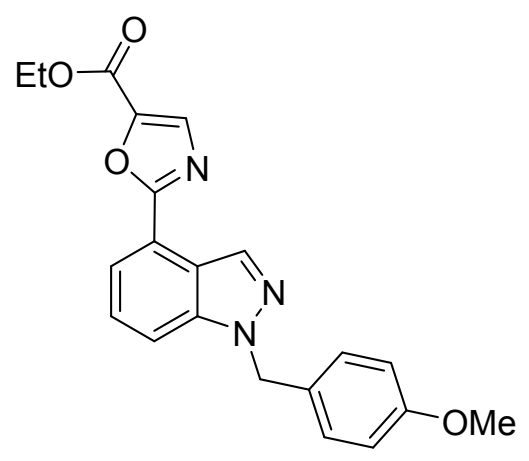

Ethyl 2-(1-(4-methoxybenzyl)-1/-indazol-4-yl)oxazole-5-carboxylate (25)

4-Bromo-1-(4-methoxybenzyl)-1/-indazole (18) (225 mg, $0.709 \mathrm{mmol}), \mathrm{Pd}(\mathrm{OAc})_{2}(8.0$

$\mathrm{mg}, 0.035 \mathrm{mmol}), \mathrm{Cs}_{2} \mathrm{CO}_{3}(693 \mathrm{mg}, 2.13 \mathrm{mmol})$, pivalic acid $(28.9 \mathrm{mg}, 0.283 \mathrm{mmol})$ and dicyclohexyl(2',6'-diisopropoxy-[1,1'-biphenyl]-2-yl)phosphine (Ruphos, $33.1 \mathrm{mg}, 0.071$

mmol) were combined in degassed PhMe (2 mL). Ethyl oxazole-5-carboxylate (3) (100

$\mathrm{mg}, 0.709 \mathrm{mmol}$ ) was added and the mixture heated at $100^{\circ} \mathrm{C}$ for $3 \mathrm{hrs}$. On cooling, the mixture was diluted with water $(20 \mathrm{~mL})$ and extracted with $\mathrm{PhMe}(2 \times 20 \mathrm{ml})$. The combined organics were evaporated to dryness. Purification by silica gel chromatography, eluting with 10-30\% EtOAc in heptane, afforded ethyl 2-(1-(4methoxybenzyl)-1 H-indazol-4-yl)oxazole-5-carboxylate (246 mg, $0.652 \mathrm{mmol}, 92 \%$ ) as a white solid. 
${ }^{1} \mathrm{H}$ NMR $\left(400 \mathrm{MHz}, \mathrm{CDCl}_{3}\right) \delta_{\mathrm{H}} 8.64(1 \mathrm{H}, \mathrm{s}), 7.99(1 \mathrm{H}, \mathrm{d}, J=7.1 \mathrm{~Hz}), 7.91(1 \mathrm{H}, \mathrm{d}, J=8.6$ $\mathrm{Hz}), 7.89(1 \mathrm{H}, \mathrm{s}), 7.39(1 \mathrm{H}, \mathrm{dd}, J=8.6,7.1 \mathrm{~Hz}), 7.31(2 \mathrm{H}, \mathrm{d}, J=8.6 \mathrm{~Hz}), 6.89(1 \mathrm{H}, \mathrm{d}, J$ $=8.6 \mathrm{~Hz}), 5.59(2 \mathrm{H}, \mathrm{s}), 4.43(2 \mathrm{H}, \mathrm{q}, J=7.1 \mathrm{~Hz}), 1.42(3 \mathrm{H}, \mathrm{t}, J=7.1 \mathrm{~Hz}) ;{ }^{13} \mathrm{C}$ NMR $(100$ $\left.\mathrm{MHz}, \mathrm{CDCl}_{3}\right) \delta_{\mathrm{C}} 163.6,159.7,157.9,149.0,141.9,135.4,129.5,127.6,125.4,124.4$ 122.8, 121.9, 118.7, 118.5, 114.3, 61.4, 57.3, 55.3, 14.3; HRMS $m / z$ calc'd for $\mathrm{C}_{21} \mathrm{H}_{20} \mathrm{~N}_{3} \mathrm{O}_{4}$ 378.1448, measured 378.1443 ; m.p. $119-122^{\circ} \mathrm{C}$.

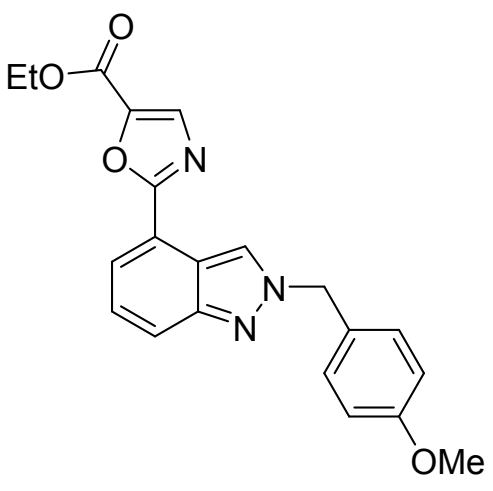

Ethyl 2-(2-(4-methoxybenzyl)-2H-indazol-4-yl)oxazole-5-carboxylate (26)

4-Bromo-2-(4-methoxybenzyl)-2H-indazole (19) (225 mg, $0.709 \mathrm{mmol}), \mathrm{Pd}(\mathrm{OAc})_{2}(8.0$ $\mathrm{mg}, 0.035 \mathrm{mmol}), \mathrm{Cs}_{2} \mathrm{CO}_{3}(693 \mathrm{mg}, 2.13 \mathrm{mmol})$, pivalic acid $(28.9 \mathrm{mg}, 0.283 \mathrm{mmol})$ and dicyclohexyl(2',6'-diisopropoxy-[1,1'-biphenyl]-2-yl)phosphine (Ruphos, $33.1 \mathrm{mg}, 0.071$ mmol) were combined in degassed PhMe (2 mL). Ethyl oxazole-5-carboxylate (3) (100 
$\mathrm{mg}, 0.709 \mathrm{mmol}$ ) was added and the mixture heated at $100^{\circ} \mathrm{C}$ for $3 \mathrm{hrs}$. On cooling, the mixture was diluted with water $(20 \mathrm{~mL})$ and extracted with PhMe $(2 \times 20 \mathrm{~mL})$. The combined organics were evaporated to dryness. Purification by silica gel chromatography, eluting with $0-25 \%$ EtOAc in heptane, afforded ethyl 2-(2-(4methoxybenzyl)-2H-indazol-4-yl)oxazole-5-carboxylate (225 mg, $0.596 \mathrm{mmol}, 84 \%)$ as a white solid.

${ }^{1} \mathrm{H}$ NMR $\left(400 \mathrm{MHz}, \mathrm{CDCl}_{3}\right) \delta_{\mathrm{H}} 8.77(1 \mathrm{H}, \mathrm{s}), 8.00(1 \mathrm{H}, \mathrm{d}, J=7.3 \mathrm{~Hz}), 7.94(1 \mathrm{H}, \mathrm{s}), 7.52$ $(1 \mathrm{H}, \mathrm{d}, J=8.3 \mathrm{~Hz}), 7.42(1 \mathrm{H}, \mathrm{dd}, J=8.3,7.3 \mathrm{~Hz}), 7.17(2 \mathrm{H}, \mathrm{d}, J=8.6 \mathrm{~Hz}), 6.83(2 \mathrm{H}, \mathrm{d}, J$ $=8.6 \mathrm{~Hz}), 5.60(2 \mathrm{H}, \mathrm{s}), 4.45(2 \mathrm{H}, \mathrm{q}, J=7.1 \mathrm{~Hz}), 3.76(3 \mathrm{H}, \mathrm{s}), 1.44(3 \mathrm{H}, \mathrm{t}, J=7.1 \mathrm{~Hz}) ;{ }^{13} \mathrm{C}$ $\operatorname{NMR}\left(100 \mathrm{MHz}, \mathrm{CDCl}_{3}\right) \delta_{\mathrm{C}} 163.4,159.3,157.8,142.2,139.8,135.4,133.9,133.9,128.5$ 126.0, 121.2, 121.0, 119.1, 114.1, 112.9, 61.5, 55.2, 52.8, 14.3; HRMS $m / z$ calc'd for $\mathrm{C}_{21} \mathrm{H}_{20} \mathrm{~N}_{3} \mathrm{O}_{4} 378.1448$, measured 378.1449 ; m. p. $127-129^{\circ} \mathrm{C}$. 


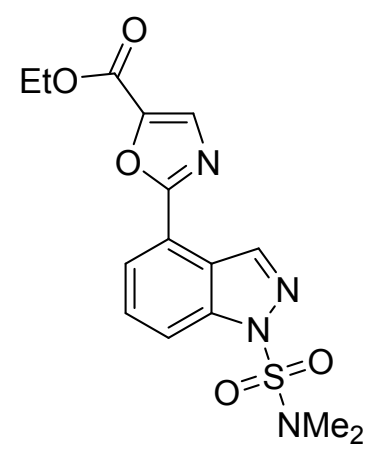

Ethyl 2-(1-(N,N-dimethylsulfamoyl)-1 H-indazol-4-yl)oxazole-5-carboxylate (29)

4-bromo- $N, N$-dimethyl-1 H-indazole-1-sulfonamide (18) (216 mg, $0.710 \mathrm{mmol}), \operatorname{Pd}(\mathrm{OAc})_{2}$

(8.0 mg, $0.035 \mathrm{mmol}), \mathrm{Cs}_{2} \mathrm{CO}_{3}(693 \mathrm{mg}, 2.13 \mathrm{mmol})$, pivalic acid $(28.9 \mathrm{mg}, 0.283 \mathrm{mmol})$

and dicyclohexyl(2',6'-diisopropoxy-[1,1'-biphenyl]-2-yl)phosphine (Ruphos, $33.1 \mathrm{mg}$,

$0.071 \mathrm{mmol}$ ) were combined in degassed PhMe (2 mL). Ethyl oxazole-5-carboxylate (3)

(100 mg, $0.709 \mathrm{mmol}$ ) was added and the mixture heated at $100^{\circ} \mathrm{C}$ for $3 \mathrm{hrs}$. On cooling,

the mixture was diluted with water $(20 \mathrm{~mL})$ and extracted with PhMe $(2 \times 20 \mathrm{~mL})$. The

combined organics were evaporated to dryness. Purification by silica gel

chromatography, eluting with $0-20 \%$ EtOAc in heptane, afforded ethyl 2-(1-(N,N-

dimethylsulfamoyl)-1 H-indazol-4-yl)oxazole-5-carboxylate (221 mg, $0.607 \mathrm{mmol}, 85 \%)$ as

a white solid. 
${ }^{1} \mathrm{H}$ NMR $\left(400 \mathrm{MHz}, \mathrm{CDCl}_{3}\right) \delta_{\mathrm{H}} 8.97(1 \mathrm{H}, \mathrm{s}), 8.22(1 \mathrm{H}, \mathrm{d}, J=8.3 \mathrm{~Hz}), 8.11(1 \mathrm{H}, \mathrm{d}, J=7.6$ $\mathrm{Hz}), 7.92(1 \mathrm{H}, \mathrm{s}), 7.62(1 \mathrm{H}, \mathrm{dd}, J=8.3,7.6 \mathrm{~Hz}), 4.44(2 \mathrm{H}, \mathrm{q}, J=7.1 \mathrm{~Hz}), 2.99(6 \mathrm{H}, \mathrm{s})$, $1.43(3 \mathrm{H}, \mathrm{t}, J=7.1 \mathrm{~Hz}) ;{ }^{13} \mathrm{C} \mathrm{NMR}\left(100 \mathrm{MHz}, \mathrm{CDCl}_{3}\right) \delta_{\mathrm{C}} 162.3,157.6,142.5,141.3,139.3$, 135.3, 128.6, 123.2, 121.5, 119.3, 116.1, 61.6, 38.8, 14.2; HRMS $\mathrm{m} / \mathrm{z}$ calc'd for $\mathrm{C}_{15} \mathrm{H}_{17} \mathrm{~N}_{4} \mathrm{O}_{5} \mathrm{~S} 365.0914$, measured 365.0917 ; m.p. $116-117^{\circ} \mathrm{C}$.

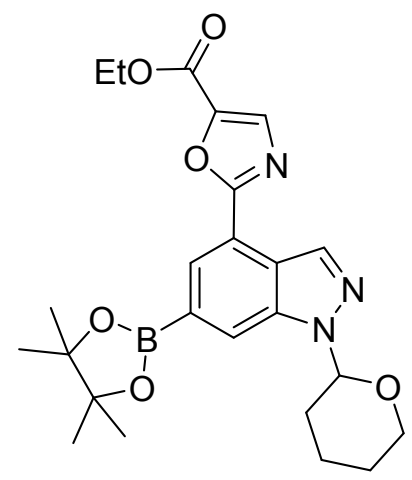

Ethyl 2-(1-(tetrahydro-2H-pyran-2-yl)-6-(4,4,5,5-tetramethyl-1,3,2-dioxaborolan-2-yl)-1 Hindazol-4-yl)oxazole-5-carboxylate (32)

Ethyl 2-(1-(tetrahydro-2 H-pyran-2-yl)-1 H-indazol-4-yl)oxazole-5-carboxylate (24) (2.00 g, $5.86 \mathrm{mmol}), 4,4^{\prime}$-di-tert-butyl-2,2'-bipyridine (39 mg, $\left.0.145 \mathrm{mmol}\right)$ and $[\operatorname{lr}(\mathrm{OMe}) \mathrm{COD}]_{2}(49$ 
$\mathrm{mg}, 0.074 \mathrm{mmol})$ were combined in degassed THF $(10 \mathrm{~mL})$. Pinacolborane $(2.38 \mathrm{~mL}$, $16.4 \mathrm{mmol}$ ) was added and the mixture heated to $70^{\circ} \mathrm{C}$ for $16 \mathrm{~h}$. The solution was cooled to $50{ }^{\circ} \mathrm{C}, \mathrm{MeOH}(20 \mathrm{~mL})$ added, and aged for $30 \mathrm{mins}$. The mixture was cooled to $20^{\circ} \mathrm{C}$, aged for a further $18 \mathrm{~h}$, and the slurry filtered, washed with $\mathrm{MeOH}(2 \times 2 \mathrm{~mL})$ and the solid dried under vacuum at $50{ }^{\circ} \mathrm{C}$ to afford ethyl 2-(1-(tetrahydro-2 $H$-pyran-2-yl)-6-(4,4,5,5tetramethyl-1,3,2-dioxaborolan-2-yl)-1 H-indazol-4-yl)oxazole-5-carboxylate (2.01 g, 4.30 mmol, $73 \%$ ) as a white solid.

${ }^{1} \mathrm{H}$ NMR $\left(400 \mathrm{MHz}, \mathrm{CDCl}_{3}\right) \delta_{\mathrm{H}} 8.79(1 \mathrm{H}, \mathrm{s}), 8.45(1 \mathrm{H}, \mathrm{s}), 8.20(1 \mathrm{H}, \mathrm{s}), 7.94(1 \mathrm{H}, \mathrm{s}), 5.86$ $(1 \mathrm{H}, \mathrm{dd}, J=9.5,2.7 \mathrm{~Hz}), 4.45(2 \mathrm{H}, \mathrm{q}, J=7.1 \mathrm{~Hz}), 4.10-4.03(1 \mathrm{H}, \mathrm{m}), 3.81(1 \mathrm{H}, \mathrm{td}, J=$ 11.0, $2.7 \mathrm{~Hz}), 2.71-2.57(1 \mathrm{H}, \mathrm{m}), 2.25-2.14(1 \mathrm{H}, \mathrm{m}), 2.12-2.04(1 \mathrm{H}, \mathrm{m}), 1.85-1.63(4 \mathrm{H}, \mathrm{m})$, $1.44(3 \mathrm{H}, \mathrm{t}, J=7.1 \mathrm{~Hz}), 1.40(12 \mathrm{H}, \mathrm{s}) ;{ }^{13} \mathrm{C} \operatorname{NMR}\left(100 \mathrm{MHz}, \mathrm{CDCl}_{3}\right) \delta_{\mathrm{C}} 163.5,157.9,142.2$ $139.8,135.5,134.6,127.1,123.1,119.9,118.4,84.8,84.4,67.7,61.5,29.5,25.0,24.9$, 22.6, 14.3; HRMS $m / z$ calc'd for $\mathrm{C}_{24} \mathrm{H}_{31} \mathrm{BN}_{3} \mathrm{O}_{6}, 468.2300$, found 468.2298; m.p. 168-170 ${ }^{\circ} \mathrm{C}$.

Note: peak for C-B not observed in ${ }^{13} \mathrm{C}$ NMR. 


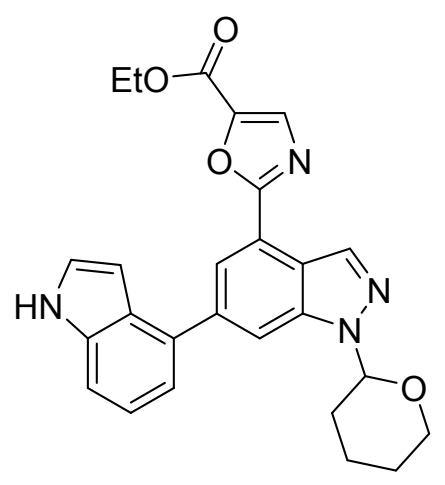

Ethyl 2-(6-(1H-indol-4-yl)-1-(tetrahydro-2H-pyran-2-yl)-1 H-indazol-4-yl)oxazole-5-

carboxylate (37)

Palladium acetate $(4.71 \mathrm{mg}, 0.021 \mathrm{mmol})$ and tricyclohexylphosphine $(11.8 \mathrm{mg}, 0.042$

$\mathrm{mmol})$ were combined in degassed 2-propanol $(2.5 \mathrm{~mL})$ and stirred for 50 mins to give a

yellow solution. This was added via cannula to a mixture of ethyl 2-(1-(tetrahydro-2 $\mathrm{H}$ -

pyran-2-yl)-6-(4,4,5,5-tetramethyl-1,3,2-dioxaborolan-2-yl)-1H-indazol-4-yl)oxazole-5-

carboxylate (32) (490 mg, $1.05 \mathrm{mmol})$, potassium bifluoride (180 mg, $2.31 \mathrm{mmol}$ ),

potassium phosphate $(267 \mathrm{mg}, 1.26 \mathrm{mmol})$ and 4-bromoindole $(0.132 \mathrm{~mL}, 1.05 \mathrm{mmol})$ in

degassed 2-propanol $(2.5 \mathrm{~mL})$ and water $(2.5 \mathrm{~mL})$ at $90^{\circ} \mathrm{C}$. After $3.5 \mathrm{hrs}$, water $(2.5 \mathrm{~mL})$

was added and the mixture was cooled to $20^{\circ} \mathrm{C}$, aged for $2 \mathrm{~h}$, filtered, and the solid

washed with 1:1 water:2-propanol $(2 \mathrm{~mL})$ and dried at $55^{\circ} \mathrm{C}$ under vacuum to afford ethyl 
2-(6-(1 H-indol-4-yl)-1-(tetrahydro-2 $H$-pyran-2-yl)-1 $H$-indazol-4-yl)oxazole-5-carboxylate (420 mg, $0.920 \mathrm{mmol}, 88 \%)$ as a grey solid.

${ }^{1} \mathrm{H}$ NMR $\left(400 \mathrm{MHz}, \mathrm{CDCl}_{3}\right) \delta_{\mathrm{H}} 8.81(1 \mathrm{H}, \mathrm{s}), 8.48(1 \mathrm{H}, \mathrm{br} \mathrm{s}), 8.41(1 \mathrm{H}, \mathrm{d}, J=1.0 \mathrm{~Hz}), 8.09$ $(1 \mathrm{H}, \mathrm{s}), 7.97(1 \mathrm{H}, \mathrm{s}), 7.49-7.45(1 \mathrm{H}, \mathrm{m}), 7.34(1 \mathrm{H}, \mathrm{s}), 7.34-7.33(1 \mathrm{H}, \mathrm{m}), 7.31(1 \mathrm{H}, \mathrm{t}, J=$ $2.8 \mathrm{~Hz}), 6.75(1 \mathrm{H}, \mathrm{s}), 5.85(1 \mathrm{H}, \mathrm{dd}, J=9.2,2.6 \mathrm{~Hz}), 4.45(2 \mathrm{H}, \mathrm{q}, J=7.1 \mathrm{~Hz}), 4.09-4.05$ (1H, m), 3.78-3.72 (1H, m), 2.65-2.56 (1H, m), 2.25-2.18 (2H, m), 1.79-1.65 (3H, m), 1.44 $(3 \mathrm{H}, \mathrm{t}, J=7.1 \mathrm{~Hz}) ;{ }^{13} \mathrm{C}$ NMR $\left(100 \mathrm{MHz}, \mathrm{CDCl}_{3}\right) \delta_{\mathrm{C}} 163.5,157.9,142.3,140.6,140.0$ $136.3,135.6,134.4,133.3,126.3,125.0,123.3,122.3,120.7,120.3,118.9,113.4,110.9$ 101.8, 85.7, 67.4, 61.5, 29.5, 25.1, 22.5, 14.3;; HRMS $m / z$ calc'd for $\mathrm{C}_{26} \mathrm{H}_{25} \mathrm{~N}_{4} \mathrm{O}_{4}$ 457.1870, measured 457.1875 ; m.p. $210-215^{\circ} \mathrm{C}$.

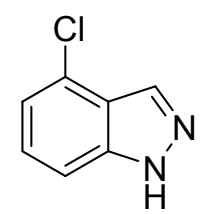

\section{4-Chloro-1 H-indazole}


Acetic anhydride (69 kg, $675.9 \mathrm{~mol})$ was added to a stirred slurry of 3-chloro-2methylaniline $(30 \mathrm{~kg}, 211.9 \mathrm{~mol})$, potassium acetate $(25 \mathrm{~kg}, 254.7 \mathrm{~mol})$ and methyltetrahydrofuran $(302 \mathrm{~L})$ at $25^{\circ} \mathrm{C}$ and then stirred for $2 \mathrm{~h}$. Isopentyl nitrite $(44.5 \mathrm{~kg}$, $379.9 \mathrm{~mol}$ ) was added to the slurry and the contents were heated to $73^{\circ} \mathrm{C}$ for $18 \mathrm{~h}$. The slurry was cooled to $20^{\circ} \mathrm{C}$, then water $(90 \mathrm{~L})$ was added, and the reaction was cooled to $5{ }^{\circ} \mathrm{C}$. Aqueous $\mathrm{NaOH}$ ( $105 \mathrm{~L}$ of a $32 \% \mathrm{w} / \mathrm{w}$ solution) was added, the solution was heated to $40{ }^{\circ} \mathrm{C}$ and stirred for $2 \mathrm{~h}$. The lower aqueous phase was removed and the organic layer washed with water $(150 \mathrm{~L})$ and then brine (18 $\mathrm{kg}$ in $90 \mathrm{~L}$ water). The organic layer was concentrated to $90 \mathrm{~L}$ by atmospheric distillation and a solvent swap to $n$-heptane performed by atmospheric distillation to a final volume of $240 \mathrm{~L}$. The slurry was cooled to $7^{\circ} \mathrm{C}$, stirred for $2 \mathrm{~h}$ and solid isolated by filtration. The cake was washed with heptane $(2$ $\times 60 \mathrm{~L})$ and dried under vacuum to give the title compound $(23.6 \mathrm{~kg}, 73 \%)$.

${ }^{1} \mathrm{H}$ NMR $\left(400 \mathrm{MHz}, \mathrm{CD}_{3} \mathrm{OD}\right) \delta_{\mathrm{H}} 8.08(\mathrm{~s}, 1 \mathrm{H}), 7.48(\mathrm{~d}, J=8.6 \mathrm{~Hz}, 1 \mathrm{H}), 7.33(\mathrm{dd}, J=7.5$, $8.4 \mathrm{~Hz}, 1 \mathrm{H}), 7.14(\mathrm{~d}, J=7.3 \mathrm{~Hz}, 1 \mathrm{H})$. 


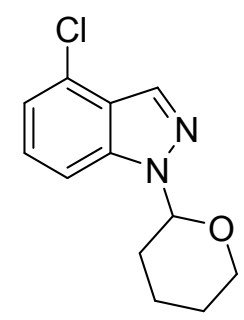

\section{4-Chloro-1-(tetrahydro-2H-pyran-2-yl)-1 H-indazole (44)}

Trifluoroacetic acid $(3.5 \mathrm{~kg}, 30.7 \mathrm{~mol})$ was added to a solution of 4-chloro- $1 /$-indazole (23 kg, $15.1 \mathrm{~mol})$ and 3,4-dihydro-2 $\mathrm{H}$-pyran $(43.1 \mathrm{~kg}, 512.7 \mathrm{~mol})$ in EtOAc (235 L). The reaction mixture was heated to $80^{\circ} \mathrm{C}$ for $4 \mathrm{~h}$, then cooled to $23^{\circ} \mathrm{C}$, triethylamine $(3.2 \mathrm{~kg}$,

$31.6 \mathrm{~mol}$ ) added and stirred for $30 \mathrm{~min}$. The reaction mixture was then solvent swapped into isopropanol using an atmospheric distillation to a final volume of $138 \mathrm{~L}$. The solution was cooled to $55{ }^{\circ} \mathrm{C}$ and water $(138 \mathrm{~L})$ was added maintaining the temperature. The solution was cooled to $42{ }^{\circ} \mathrm{C}$ and seeded $(8 \mathrm{~g})$, then cooled to $30^{\circ} \mathrm{C}$, held for $10 \mathrm{~h}$, and water (46 L) added, cooled to $18^{\circ} \mathrm{C}$, and the slurry was stirred for $2 \mathrm{~h}$. The product was filtered off, washed with 6:1 v/v water/2-propanol $(2 \times 46 \mathrm{~L})$ and dried under vacuum at $50{ }^{\circ} \mathrm{C}$ to give the title compound $(28.8 \mathrm{~kg}, 80.7 \%)$. 
${ }^{1} \mathrm{H}$ NMR $\left(400 \mathrm{MHz},\left(\mathrm{DC}_{3}\right)_{2} \mathrm{SO}\right) \delta_{\mathrm{H}} 8.18(\mathrm{~s}, 1 \mathrm{H}), 7.74(\mathrm{~d}, J=8.5 \mathrm{~Hz}, 1 \mathrm{H}), 7.42(\mathrm{dd}, J=7.5$,

$8.4 \mathrm{~Hz}, 1 \mathrm{H}), 7.27(\mathrm{~d}, J=7.3 \mathrm{~Hz}, 1 \mathrm{H}), 5.88(\mathrm{dd}, J=2.5,9.5 \mathrm{~Hz}, 1 \mathrm{H}), 3.90-3.85(\mathrm{~m}, 1 \mathrm{H})$, 3.79-3.70 (m, 1H), 2.44-2.35 (m, 1H), 2.07-1.95 (m, 2H), 1.81-1.69 (m, 1H), 1.64-1.53 (m, $2 \mathrm{H})$. 